\title{
Is There a Correlation between Academic Achievement and Behavior in Mainland Chinese Students?
}

\author{
Haibin Li (Corresponding author) \\ Faculty of Education and Social Work, University of Sydney \\ NSW2006, Australia \\ Tel: 61-2-9036-5300Ｅ-mail: haibin.li@edfac.usyd.edu.au \\ Derrick Armstrong \\ Professor in Faculty of Education and Social Work, University of Sydney \\ NSW2006, Australia \\ Tel: 61-2-9351-3517Ｅ-mail: derrick.armstrong@usyd.edu.au
}

\section{Acknowledgements}

The authors would like to thank Dr Richard Walker for his helpful comments on an earlier draft of this paper.

\begin{abstract}
Academic achievement and behavior have been demonstrated to be clearly associated in the western literature. However, few studies concerning these relationships have been conducted in Asian contexts. This study attempts to provide empirical evidence from the east to complement the wealth of research in the west and examine whether the findings in the west can be generalized to Asian cultures. Data were collected from 636 students in year 11 at four Chinese high schools. Students' academic achievement was assessed using their grade point average (GPA). Behavior was assessed from students' reports and three aspects: truancy; substance use and antisocial behavior were focused on. Correlation analysis indicated that some problematic behaviors (e.g., truancy, stealing, receiving, and vandalism) were not related to GPA and some problematic behaviors (e.g., drinking, smoking and violence) were weakly, negatively but significantly related to students' GPA. Moreover, high academic achievement was negatively but weakly related to a number of problematic behaviors. There was also no statistically significant difference in the strength of the relationship for males and females.
\end{abstract}

Keywords: Chinese adolescents, Academic achievement, Behavior, Sex difference

\section{Introduction}

Recently, the dramatic increase in the prevalence of problematic behaviors during adolescence has broken the traditional view that academic success is the only standard in assessing good students. In many western countries, not only students' school performance, but also their behavior has been given considerable public attention. For example, Masten and her associates (1995) proposed that for school-age children, high academic achievement and behavioral competence should be regarded as two important indicators for their success. Therefore, to explore the relationship between students' behavior and academic achievement has important implications for developing effective intervention programs.

The relationship between behavior and academic achievement has been demonstrated in a number of studies. The balance of evidence from long-standing research suggests that problematic behavior and academic failure are clearly associated both concurrently and predictively in western literature (see Hinshaw, 1992 for a review). Generally, the link between the two variables is explained from two points of view.

The first is the "common-developmental-antecedent" explanation. People who hold this view think that the association 
between academic achievement and behavior problems is influenced by either genetic/intrapersonal factors (Gayan \& Olson, 1999; Rhee \& Waldman, 2002) or environmental antecedents (Ary, Duncan, Duncan, \& Hops, 1999; Richman, Stevenson, \& Graham, 1982).

An alternative view is the "causal relation" explanation. Three plausible models have been proposed to explain the causal association between school performance and problematic behavior. The first is that underachievement leads to problematic behavior (McGee, Williams, Share, Anderson, \& Silva, 1986; Stevenson, Richman, \& Graham, 1985). Thus, low academic achievement leads to a loss of self-esteem, low commitment to school, and frustration, which in turn, results in delinquency, and antisocial behavior. The second is that problem behavior precedes and causes underachievement (Dishion, 1990; Jorm, Share, Matthews, \& Maclean, 1986; Sanson, Prior, \& Smart, 1996). This mechanism is explained as follows. The amount of time children are engaged in meaningful learning activities is reduced due to their time spent acting out or being disciplined for aggressive behavior. Additionally, aggressive children may also develop negative relationships with teachers and peers or negative feelings about school, and as a consequence be less inclined to exert effort on academic work (Arnold, 1997; Wentzel \& Asher, 1995). The third is that each domain leads to the other (Arnold, 1997; McMichael, 1979), in other words, the causal relations between school performance and problematic behavior are bidirectional instead of unidirectional. This view holds that when poor learners become increasingly frustrated, their antisocial behavior increases, which in turn disrupts the processes of learning, which then creates more antisocial problems, and so on.

From this evidence, it might be predicted that high academic achievement would be clearly associated with lack of problematic behavior and either "common-developmental-antecedent" or the "causal relation" mechanisms should be applicable to explaining the relation between the two variables. However, two news reports (Kang \& Saar, 1996; Seo, 1995) have argued that some Asian students in the USA involved in violence had attained top grade-point averages, which indicates that high academic achievement is not strongly associated with a lower level of problematic behavior among Asian Pacific Islander American youth compared with other racial and ethnic groups of youth.

Differences between western and eastern culture may lead to different outcomes in the relations between academic achievement and behavior. The studies reported in the newspaper articles had entirely male samples and this may have skewed the findings.

In Chinese culture, popular maxims such as "to be a scholar is to be the top of society" and "through studying you will gain beautiful girls and houses made of gold" drive most Chinese to believe that, by excelling academically, they will gain success and fortune will follow. Additionally, the goals of Chinese school education are to help students develop well in behavior, as well as academic achievement (Chen, Rubin, \& Li, 1997; The goals of school education, 1970). However, due to the current limited opportunities to receive higher education, academic competition is extremely strong in schools, especially in high schools. Consequently, Chinese students are pushed very hard to excel in school by their families and schools. If students are doing well in school, their parents and teachers think everything is OK. On the contrary, if students fail to achieve high standards in academic achievement, they are often regarded as abnormal and problematic by parents, teachers, and peers ( $\mathrm{Wu} \&$ Tseng, 1985). As a result, students' academic competence has received much attention while any behavioral problems have been largely neglected in practice.

Too few studies concerning the association of academic achievement and behavior have been conducted in Asian contexts. Therefore, there is a pressing need to examine the problem from this perspective and provide empirical evidence from the east to complement the wealth of research in the west. In this study, we especially concentrate on adolescence because this is the developmental stage during which problematic behavior peaks in onset, prevalence, and incidence (Moffitt, Caspi, Rutter, \& Silva, 2001) and when academic competition is strong.

Moreover, we want to examine whether gender plays a role in the relation between achievement and behavior. In the literature, girls have long been demonstrated by criminologists (e.g., Kruttschnitt, 1994) or psychologists (e.g., Bettencourt \& Miller, 1996) to have higher academic competence and be less violent than boys at every age. This sex difference could be interpreted as meaning that females lack the underlying motivation or capacity for violence that men have (Moffitt, Caspi, Rutter, \& Silva, 2001). However, as to whether gender mediates the relationship between academic achievement and behavior, there is a key debate. Two studies by Sanson et al. (1996) and Willcutt \& Pennington (2000) showed the relation was significantly stronger for boys than girls. This result is in contrast to an earlier report. For instance, Stevenson, Richman, and Graham (1985) indicated no difference between boys and girls. These few, inconsistent findings do not provide sufficient evidence for making predictions about gender and so demand further evidence.

Prompted by the newspaper articles mentioned above and based on the gaps in the literature, two main hypotheses were derived:

1). high academic achievement and behavioral competence are weakly related in the Chinese context;

2). relationship between achievement and behavior is mediated by gender. 


\section{Method}

\subsection{Participants}

Adolescents were recruited from four high schools, situated in three different districts (big city, small city and rural area) in Jiangsu Province, eastern China. Participants were 636 students (375 boys, 260 girls, and 1 case sex unspecified) enrolled in the 11 th grade. These students ranged in age from 15 years to 19 years (mean $=16.8, \mathrm{SD}=0.788$ ). Jiangsu province was selected because the area has a high population density and resident average annual incomes are ranked in the middle level of the country, and polarization of rich and poor is quite large. Our sample of parents was socio-economically mixed, ranging from the very affluent to those at the lower end of the socio-economic scale. Of all participants, $72 \%$ of them were from families in which parents were workers or peasants and $28 \%$ were from professional families in which one or both parents were teachers, doctors, or officials. Seventy-six percent of the students reported one or both of the parents had an educational level of high school or below.

\subsection{Measures}

Academic Achievement. Students' academic achievement was assessed using their grades in the major subjects (e.g., Chinese, Maths, and English). The average grade (e.g., A, B, C, D, or F) of the first semester mid-term examination was taken directly from the school records. Grades were coded on a continuous scale ranging from A (4) to F (0), with $\mathrm{M}=1.56, \mathrm{SD}=1.211$, and range $=0$ to 4 .

Behavior. Behaviors were measured by self-reports. According to Moffitt, Caspi, Rutter, and Silva (2001), self-reports from adolescents are reliable and valid while in adolescence much antisocial behavior is always concealed from other reporters (e.g., parents and teachers). Students completed a 10-item behavior inventory originally from the On Track Youth Lifestyles Surveys (Armstrong et al., 2005). The 10 items cover three categories of problematic behaviors: truancy, use of substance (alcohol and smoking), and antisocial behaviors (stealing, receiving, attacking and vandalizing). Students were asked how many times in the previous year they exhibited these antisocial behaviors and how many times in the last four weeks they involved truancy and use of substance. Responses options for these items were "none" (0), "once" (1), twice (2), "3-5 times" (3), 6 or more times (4). A Chinese translation was made by a bilingual translator (English and Mandarin) and sent to another translator for back translation. The bilingual translator also verified, subsequently, that the final Chinese version conformed to the original English version. Responses from the questions in this study were recoded into a binary response. "0" was coded if the respondent had never committed the act, and "1" was coded if the student had done it once or more.

\subsection{Procedure}

The research received ethical approval from the University's Human Ethics Review Committee. Participants in the study were completely voluntary. Before the study was conducted, students and their parents/guardians were provided with a written summary of the research procedure, its benefits, harms and risks through a letter with a permission form enclosed. Only those students and their parents/guardians who both signed the consent forms were included in the study. Students were also fully informed about the study and were able to withdraw from the process at any stage with no penalty to them.

\subsection{Analysis}

Preliminary analyses included descriptive statistics' comparisons of the two main variables (GPA and behavior) across three samples (full sample, males and females). In the next step, relationships among behavior variables and between academic achievement and behavior were tested via correlation analysis. In the study, 18 questionnaires had missing data and these were excluded.

\section{Results}

In order to examine the relationship between school attainment and behavior, three broad aspects of the results were investigated. The first concerned the levels of academic achievement and the total number of behavior problems. The second concerned interrelations among GPA, aspects of truancy, substance use, and antisocial behaviors. The third concerned the correlation between GPA and number of problematic behaviors in three different groups (males, females and total sample).

\section{INSERT TABLE 1 ABOUT HERE}

\subsection{Profiles of students' academic achievement and problematic behaviors}

Table 1 presents means and standard deviations for each variable (GPA and the total number of behavior problems) for the boys and girls separately. The mean GPA mark in total students $(n=636)$ is 1.56 with the high score being 4 and both boys and girls presented less than 1 problematic behavior (mean=.55). Compared with girls, boys had lower scores on GPA and higher scores on problematic behavior. 


\subsection{Intercorrelations among variables}

Intercorrelations among the GPA and behavior variables are presented in Table 2. Generally, the correlations between academic achievement scores and the 7 problematic behavior scores were weak $(\mathrm{r}<.30)$ with most at or near zero level. The associations indicate that the level of problematic behavior engaged in by adolescents generally has little relationship with their academic achievement. These correlations also indicate negative relations between GPA and these problematic behaviors. The one exception was students' GPA and their stealing behaviors, with a correlation of $r$ $=0$, which indicated that there was completely no relation between the two variables. In addition, four of these coefficients were statistically significant $(\mathrm{p}<0.01)$, and it tells us the relations did not happen by chance.

From Table 2, it can be observed that the coefficients among problematic behavior variables ranged from .03 to .53 with most at a significant level $(\mathrm{p}<0.01)$. It implied that these problematic behaviors are low to moderate correlated positively with each other.

\section{INSERT TABLE 2 ABOUT HERE}

\subsection{Relationship between problematic behavior and academic achievement}

Bivariate correlation was used to explore the relationship between students' achievement (GPA) and number of problematic behaviors in three separate groups (full sample, male and female). From Table 3, it can be seen that there was weak, negative and significant correlation between achievement and behavior problem $(\mathrm{r}=-.17, \mathrm{n}=620, \mathrm{p}<.01)$, with high achievement being weakly associated with low problematic behaviors.

\section{INSERT TABLE 3 ABOUT HERE}

Inspecting the two separate groups (male and female), the correlation between GPA and total problematic behaviors for males was $\mathrm{r}=-.12$, while for females it was slightly higher, $\mathrm{r}=-.17$. The coefficients -.12 and -.17 suggested that GPA and problematic behaviors were negatively and low correlated at a significant level. To assess the probability that difference in the correlations observed for the two groups would occur as a function of a sampling error, the authors convert the $r$

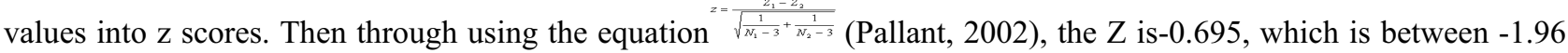
and +1.96 . Therefore, there is no statistically significant difference in the strength of the relationship for males and females.

\section{Discussion}

The first hypothesis was that competencies in academic and behavioral domains are weakly related in Chinese context. The second hypothesis was that the association between school performance and adolescent behaviors is moderated by gender. The findings of the current study provide support for the first hypothesis but reject the second hypothesis.

The results of the correlational analyses indicate that, even though the relationships between high achievement and low problematic behavior were statistically significant, the correlations were typically quite low. This means that students with high academic scores may not exhibit behavioral competence. Additionally, the findings are clear-cut in showing that girls had higher scores on GPA and less problematic behaviors than boys, which is similar to earlier results (e.g., Moffitt, Caspi, Rutter, \& Silva, 2001; Rutter et al., 2004). However, there is no statistically significant difference in the strength of the relationship between males and females.

The findings reject the notion that the reciprocal co-existing relationship between high academic achievement and good behaviors found in the west is present among Chinese adolescents. The results did not mesh well with the conclusion from western literature (e.g., Hinshaw, 1992). On the contrary, they are consistent with the news reports about Asian Pacific Islander American youth (e.g., Kang \& Saar, 1996; Seo, 1995). The study provides evidence that compared with the western counterparts, Asian adolescents can achieve good academic performance in school, but at the same time, exhibit behavior problems. It could be explained that in China, academic competition is extremely strong for high school students. Under the high pressure, they have no time to conduct those problematic behaviors. Sometimes, engaging in those problematic behaviors was regarded as a way to relieving pressure from examination for all students. However, due to the limited samples, the conclusion can only be confirmed at a very minimal level.

The results yield useful information that contributes to theoretical knowledge on the link between academic achievement and behavior. In this study, we can not determine any causal relationship between the two variables. However, these correlational results force us to reflect on the "common-developmental-antecedent" explanation in interpreting the relationship between academic achievement and problematic behavior (e.g., Hinshaw, 1992). The 3\% variance $(\mathrm{r}=.17)$ between the two variables indicated that few or no common factors influence the academic and behavioral outcomes.

The study also found that males had more problematic behavior than females in the Chinese context. However, there is no difference in the relation between high GPA and behavioral competence for males and females. If we excluded the fact that female high school students are not representative of the general female population, then doubt is cast on the 
theory that females have less motivation or capacity for violence than men (Moffitt, Caspi, Rutter, \& Silva, 2001).

\subsection{Strengths and limitations of the design of the study}

Some strengths of this study need to be mentioned. Firstly, the results of the present study are important because relatively few existing studies have addressed this relationship in Asian cultures or among Asian youth. Taking a prospective approach has helped delineate the relation between high academic achievement and good behaviors. Secondly, though the sample was not a representative one of Chinese adolescents, it is based on a mixed SES sample. Results can be generalized to most Chinese high school adolescents.

Despite these advantages, a few limitations should be taken into consideration when interpreting the findings. First, the correlational nature of the research precludes conclusions concerning a causal prediction between the two variables. Longitudinal studies may reveal a recursive effect between behaviors and school achievement in one or the other direction. Second, in the present study, adolescents' family characteristics and problematic behavior were self-reported. The mono-informant character of that information could have skewed the relations between school attainment and behavior.

\subsection{Further research and applications to practice}

The findings yield useful information that contributes to theoretical knowledge on the relationship between competencies in academic and social domains and also has applications for work with adolescents in preventive intervention programs. The weak link between high GPA and good behavior request educators to consider why some students with high achievement have behavior problem. Failure to account for the question may lead to inappropriate or inefficient training programs. In addition, the findings indicated that the relationship is weak and there is no sex difference in the association between academic attainment and problematic behavior. This suggests that, for both boys and girls, it is impractical to improve academic achievement through reducing behavior problems, or vice versa.

This study suggests several directions for future research. Firstly, a longitudinal study should be conducted to identify any causal relationship between academic achievement and behavioral outcomes in the Chinese context. Such evidence would highlight whether the two variables are related both concurrently and predictively, and then allow a comparison with the western results systematically. Secondly, the data for behavior variables in this study were obtained from students' self-reports. If data can be obtained by multimethods and from multisources (e.g., the participants themselves, peers, teachers and parents), some common method and source biases that can inflate relationships could be remedied. Thirdly, we need to design a study to systematically explore why academic attainment and behavior among Chinese youth are only weakly linked and reconsider what kind of underlying factors play determinant roles in promoting academic or behavioral competencies in the Chinese context. For example, a qualitative research such as case studies or interview can be designed for students in trouble to ascertain what other factors need to be included, and then path analysis or structural equation modeling could be used to analyze the correlation among these required variables. Lastly, a recent study (Choi, 2007) found that, if age, gender, parental education and earlier problematic behaviors are controlled, generally academic achievement is a significant predictor of the problematic behaviors regardless of one's race or ethnicity. If this is the case, future research needs to control the variables mentioned above and examine whether academic achievement is a significant predictor of the problematic behavior among Chinese adolescents.

\section{References}

Armstrong, D., Hine, J., Hacking, S., Armaos, R., Jones, R., Klessinger, N., et al. (2005). Children, risk and crime: the on track youth lifestyles surveys. London: Home Office.

Arnold, D. H. (1997). Co-occurrence of externalizing behavior problems and emergent academic difficulties in young high-risk boys: A preliminary evaluation of patterns and mechanisms. Journal of Applied Developmental Psychology, 18, 317-330.

Ary, D. V., Duncan, T. E., Duncan, S. C., \& Hops, H. (1999). Adolescent problem behavior: The influence of parents and peers. Behaviour Research and Therapy, 37, 217-230.

Bettencourt, B. A., \& Miller, N. (1996). Gender differences in aggression as a function of provocation: A meta-analysis. Psychological Bulletin, 119, 422-447.

Chen, X., Rubin, K. H., \& Li, D. (1997). Relation between academic achievement and social adjustment: Evidence from Chinese children. Developmental psychology, 33(3), 518-525.

Choi, Y. (2007). Academic achievement and problem behaviors among Asian Pacific Islander American adolescents. Journal of Youth Adolescence, 36, 403-415.

Dishion, T. J. (1990). The family ecology of boys' peer relations in middle childhood. Child Development, 61, 874-892.

Gayan, J., \& Olson, R. K. (1999). Reading disability: Evidence for a genetic etiology. European Child \& Adolescent 
Psychiatry, 8, 52-55.

Hinshaw, S. P. (1992). Externalizing behavior problems and academic underachievement in childhood and adolescence: Causal relationships and underlying mechanisms. Psychological Bulletin, 111, 127-155.

Jorm, A. F., Share, D., Matthews, R., \& Maclean, R. (1986). Behaviour problems in specific reading retarded and general backward children: A longitudinal study. Journal of Child Psychology and Psychiatry, 27, 33-43.

Kang, K. C., \& Saar, M. (1996). Asian gangs rise strikes a paradox; Violence: Members often come from affluent homes and do well in schools. Los Angeles times, p. 3.

Kruttschnitt, C. (1994). Gender and interpersonal violence. In A. J. Reiss, Jr. \& J. A. Roth (Eds.), Understanding and preventing violence: Vol.3. Social influences (pp. 293-376). Washington, DC: National Academy Press.

Masten, A. S., Coatsworth, J. D., Neemann, J., Gest, S. D., Tellegen, A., \& Garmezy, N. (1995). The Structure and Coherence of Competence from Childhood Through Adolescence. Child Development, 66(6), 1635-1659.

McGee, R., Williams, S., Share, D., Anderson, J., \& Silva, P. (1986). The relationship between specific reading retardation, general reading backwardness and behavioural problems in a large sample of Dunedin boys: A longitudinal study from five to eleven years. Journal of Child Psychology and Psychiatry, 27, 597-610.

McMichael, P. (1979). The hen or the egg? Which comes first: Antisocial emotional disorders or reading disability? British Journal of Educational Psychology, 49, 226-238.

Moffitt, T. E., Caspi, A., Rutter, M., \& Silva, P. (2001). Sex differences in antisocial behaviour: Conduct disorder, delinquency, and violence in the Dunedin longitudinal study. Cambridge, UK: Cambridge University Press.

Pallant, J. (2002). SPSS survival manual: A step by step guide to data analysis using SPSS for Windowa (Versions 12-14). Sydney: Allen \& Unwin.

Rhee, S. H., \& Waldman, I. D. (2002). Genetic and environmental influences on antisocial behavior: A meta-analysis of twin and adoption studies. Psychological Bulletin, 128, 490-529.

Richman, N., Stevenson, J., \& Graham, P. J. (1982). Preschool to school: A behavioral study. San Diego, CA: Academic Press.

Rutter, M., Caspi, A., Fergusson, D., Horwood, L. J., Goodman, R., Maughan, B., et al. (2004). Sex differences in developmental reading disability: New findings from 4 epidemiological studies. Journal of American Medical Association, 291, 2007-2012.

Sanson, A., Prior, M., \& Smart, D. (1996). Reading disabilities with and without behaviour problems at 7-8 years: Prediction from longitudinal data from infancy to 6 years. Journal of Child Psychology and Psychiatry, 37, 529-541.

Seo, D. (1995). Some 'A' students lead dual lives as gang-bangers: The Asian American says they are torn between values of parents, friends. Some engage in serious crime. Los Angeles times, p. 1.

Stevenson, J., Richman, N., \& Graham, P. (1985). Behaviour problems and language abilities at three years and behavioural deviance at eight years. Journal of Child Psychology and Psychiatry, 26, 215-230.

The goals of school education. (1970). Red Flag, 1(1).

Wentzel, K. R., \& Asher, S. R. (1995). The academic lives of neglected, rejected, popular, and controversial children. Child Development, 66, 754-763.

Willcutt, E. G., \& Pennington, B. F. (2000). Psychiatric comorbidity in children and adolescents with reading disability. Journal of Child Psychology and Psychiatry, 41, 1039-1048.

Wu, D. Y. H., \& Tseng, W. (1985). Introduction: The characteristics of Chinese culture. In W. Tseng \& D. Y. H. Wu (Eds.), Chinese culture and mental health. (pp. 3-13). New York: Academic Press. 
Table 1. Profiles of Chinese adolescent achievement and behaviours

\begin{tabular}{cccccccc}
\hline & \multicolumn{2}{c}{ Grade Point Average (GPA) } & & \multicolumn{3}{c}{ Total Number of Problematic Behaviors } \\
\cline { 2 - 3 } & Full Sample & Males & Females & & Full Sample & Males & Females \\
\hline $\mathrm{N}$ & 636 & 375 & 260 & & 620 & 365 & 255 \\
Mean & 1.56 & 1.39 & 1.79 & & .55 & .78 & .22 \\
SD & 1.21 & 1.20 & 1.20 & & .95 & 1.09 & .54 \\
Range & $0-4$ & $0-4$ & $0-4$ & & $1-7$ & $1-7$ & $1-7$ \\
\hline
\end{tabular}

Table 2. Intercorrelations among variables

\begin{tabular}{|c|c|c|c|c|c|c|c|c|}
\hline Variables & 1 & 2 & 3 & 4 & 5 & 6 & 7 & 8 \\
\hline 1.GPA & - & & & & & & & \\
\hline 2.Truancy & -.08 & - & & & & & & \\
\hline 3.Alcohol & $-.14 * *$ & $.26^{* *}$ & - & & & & & \\
\hline 4.Smoking & $-.29 * *$ & $.12 * *$ & $.38 * *$ & - & & & & \\
\hline 5.Stealing & .00 & .05 & $.17 * *$ & $.15^{* *}$ & - & & & \\
\hline 6.Receiving & -.01 & $.15^{* *}$ & $.15 * *$ & $.19 * *$ & $.53 * *$ & - & & \\
\hline 7.Violence & $-.19 * *$ & $.10^{* *}$ & $.22 * *$ & $.31 * *$ & $.21 * *$ & $.18 * *$ & - & \\
\hline 8.Vandalism & $-.08 * *$ & .03 & $.20 * *$ & $.17^{* *}$ & $.31 * *$ & $.29 * *$ & $.34 * *$ & - \\
\hline
\end{tabular}

Note 1. GPA=grade point average in three subjects (Chinese, Math, and English).

2. ** $\mathrm{p}<0.01$ (2-tailed), ${ }^{*} \mathrm{p}<0.05$ (2-tailed).

Table 3. Summary of correlations highlighting the relationship between Chinese adolescents' achievement and number of problematic behaviors.

\begin{tabular}{cccc}
\hline & \multicolumn{2}{c}{ Total Number of Problematic behaviors } \\
\cline { 2 - 4 } & Full Sample & Males & Females \\
\hline Grade Point Average (GPA) & & & \\
\hline Full Sample(n=620) & $-.17 * *(.000)$ & $-.12 *(.026)$ & $-.17 * *(.006)$ \\
Males $(\mathrm{n}=365)$ & & & - \\
Females $(\mathrm{n}=255)$ & & & \\
\hline
\end{tabular}

Note Correlations in this table are tested using Pearson's $r$.

$* \mathrm{p}<.05 ; \quad * * \mathrm{p}<.01$ 